\title{
BMJ Open Does intake of bread supplemented with wheat germ have a preventive role on cardiovascular disease risk markers in healthy volunteers? A randomised, controlled, crossover trial.
}

\author{
André Moreira-Rosário, ${ }^{1,2}$ Helder Pinheiro, ${ }^{3,4}$ Cláudia Marques, ${ }^{1,3}$ \\ José António Teixeira, ${ }^{5}$ Conceição Calhau, ${ }^{1,3}$ Luís Filipe Azevedo ${ }^{1,2}$
}

To cite: Moreira-Rosário A, Pinheiro H, Marques C, et al. Does intake of bread supplemented with wheat germ have a preventive role on cardiovascular disease risk markers in healthy volunteers? A randomised, controlled, crossover trial.. BMJ Open 2019;9:e023662. doi:10.1136/ bmjopen-2018-023662

- Prepublication history and additional material for this paper are available online. To view these files, please visit the journal online (http://dx.doi org/10.1136/bmjopen-2018023662).

Received 20 April 2018 Revised 27 August 2018 Accepted 19 0ctober 2018

Check for updates

(c) Author(s) (or their employer(s)) 2019. Re-use permitted under CC BY-NC. No commercial re-use. See rights and permissions. Published by BMJ.

For numbered affiliations see end of article.

Correspondence to André Moreira-Rosário; andrerosario@med.up.pt

\section{ABSTRACT}

Objective Intake of whole grains is associated with a reduced risk of cardiovascular disease (CVD). This evidence is also strong for bran alone, but findings about germ are conflicting. Our aim was to elucidate the role of germ in primary prevention of cardiovascular events, and therefore, a staple food was selected for $6 \mathrm{~g}$ of germ supplementation. This corresponds to sixfold increase in the global mean consumption of germ, while preserving the sensory proprieties of refined bread which is crucial for consumer's acceptance.

Design Randomised, double-blinded, crossover, controlled clinical trial with 15-week follow-up comprising a 2-week run-in, two intervention periods of 4 weeks each and a 5-week washout period.

Setting A single centre in the north of Portugal.

Participants 55 eligible healthy adults (mean age of 34 years and body mass index between 19 and $38 \mathrm{~kg} / \mathrm{m}^{2}$ ) were randomly assigned.

Interventions The study consisted of two intervention periods including daily intake of refined wheat bread enriched with $6 \mathrm{~g}$ of wheat germ and control (non-enriched bread).

Outcomes Changes in fasting cholesterol and triglycerides, fasting and postprandial glucose, insulin sensitivity and $\mathrm{C}$ reactive protein.

Results We observed no significant effect of daily intake of wheat germ on cholesterol and triglycerides levels, on postprandial glucose response and on insulin sensitivity. Incremental area under curve glucose and homeostasis model assessment for insulin resistance did not change, suggesting that $6 \mathrm{~g}$ of wheat germ have no effect on glucose metabolism. No effect was also observed in the subgroup of participants who complied with the protocol $(n=47)$.

Conclusions The absence of alterations on lipid and glucose profiles suggests that germ up to $6 \mathrm{~g} /$ day may have no preventive effect on CVD risk. However, it is important to investigate other food vehicles that can accommodate higher doses of wheat germ in future studies.

Trial registration number NCT02405507.
Strengths and limitations of this study

- This study followed the best practices for designing, conducting and reporting clinical trials to support health claims on food products, namely random allocation, double blinding, reporting methods to measure and maximise compliance.

- We used validated outcomes which are considered beneficial physiological effects for human health.

- To the best of our knowledge, this is the largest study to assess the impact of germ intake in human subjects.

- Although compliance was high, it is uncertain whether this is due to over-reporting, since there is no biomarker for wheat germ intake.

- A longer intervention period would be desired for evaluating an effect on lipoprotein cholesterol, nonetheless could have a major impact on loss to follow-up in this crossover study.

\section{INTRODUCTION}

Cardiovascular diseases (CVD) remain the leading cause of mortality in the world, having accounted for 15 million of deaths in 2015. ${ }^{1}$ Risk factors for CVD are well-identified and they include smoking, type 2 diabetes or high glucose levels, hypertension and elevated cholesterol levels. ${ }^{2}$ Diet is therefore an important modifiable risk factor for CVD and, within the diet, some dietary components may have an important preventive role. ${ }^{3-5}$ In this regard, cereal whole grains are a promising protective measure due to emerging evidence of an inverse association between their intake and CVD risk as shown by recent systematic reviews and meta-analyses. ${ }^{5-8}$ The role of whole grains in reducing CVD risk is broader: (1) by improving glucose metabolism through better postprandial glucose and insulin responses ${ }^{9}$; and (2) by reductions in 
plasma cholesterol levels. ${ }^{10}$ The health benefits associated with whole grains intake seem to be mediated by their high content in plant-derived redox-active compounds that may activate antioxidant pathways and thereby have anti-inflammatory proprieties. ${ }^{11}$

Cereal whole grains are distinguished from refined grains by the presence of bran and germ fractions. These two fractions accumulate higher amounts of protective bioactive compounds, such as fibres, micronutrients, vitamins and phytochemicals. CVD prevention has been associated with bran intake, ${ }^{72-15}$ but findings about germ are conflicting. ${ }^{716}$ Recent systematic reviews addressing prospective studies reported no inverse association between germ intake and CVD risk, in contrast with prior clinical trials involving high-risk groups. But these two types of studies report different amounts of germ intake. The meta-analyses of the prospective studies reported a low germ intake $(1 \mathrm{~g}$ /day average ranging from 0.2 to $2.9 \mathrm{~g}$ /day), ${ }^{17-19}$ while the intervention studies used a daily supplementation of 20 or $30 \mathrm{~g} /$ day during the 4-week period. Specifically, these clinical trials showed that intake of raw wheat germ can reduce cholesterol and triglycerides in rats ${ }^{20-22}$ and also in hypercholesterolaemic and hypertriglyceridaemic humans. ${ }^{23} 24$

Dietary guidelines around the world recommend $85 \mathrm{~g}$ daily intake of whole grains, ${ }^{25}$ which contains nearly $2.6 \mathrm{~g}$ of germ. However, their daily consumption is far below the recommendations and consumers prefer highly refined products. ${ }^{26-28}$ In fact, supplementation with whole-grain ingredients is therefore an elegant way to overcome consumer's preferences while it contributes for public health, as long as these ingredients are indeed beneficial. Clarification of the physiological effects of germ is needed. However, fortification of food products with germ is challenging because germ becomes rancid very rapidly due to high content in unsaturated lipids together with lipases and lipoxygenases, ${ }^{29-31}$ and it also negatively affects the sensory properties of the final food product. $^{32}$ Thus, in order to address these specificities, germ stabilisation ${ }^{33}$ is necessary immediately after milling to inhibit enzymatic rancidity, while the percentage of germ in the final product should be tested whether longterm consumer acceptance is desired.

In this context, we designed a randomised, doubleblinded, crossover, controlled clinical trial targeting the general population, in order to evaluate the physiological and metabolic effects of germ intake in a dose higher than the amount reported in the previous prospective studies, wherein no preventive CVD effect was demonstrated. The impact on CVD metabolic risk factors of daily consumption of $100 \mathrm{~g}$ of refined wheat bread enriched with $6 \mathrm{~g}$ of wheat germ during 4 weeks is here presented.

\section{METHODS}

\section{Participants}

Fifty-five healthy volunteer subjects were recruited from the Porto metropolitan area in northern Portugal through public advertisements in the University and Faculty websites and in online newspapers. The detailed study protocol has been previously described. ${ }^{34}$ Briefly, volunteers were invited to visit our Research Unit (CINTESIS) for a physical examination and a brief questionnaire about their medical history and background diet in order to check their eligibility to participate in the study. Inclusion criteria included subjects aged 18 to 60 years old, non-diabetics and non-smokers. The exclusion criteria included the use of medication/dietary supplements, potentially interfering with this trial, not willing to avoid prebiotics and probiotics for the duration of the study, and change of dietary habits within the 4 week prior to screening (for instance, to start a diet high in fibre). In the protocol manuscript, we described sample size calculations. Briefly, we calculated the sample size for each individually primary outcome, taking into account the difference in mean change from baseline and the respective SD. The sample size of the study was determined by the outcome that required the highest number of participants. Accordingly, 40 participants were required to allow for an $80 \%$ power and $95 \%$ confidence level. ${ }^{34}$

The study protocol was approved, and all participants provided written informed consent. The clinical trial was conducted from June 2015 to October 2016, in accordance with ethical principles of the Declaration of Helsinki, international law and Good Clinical Practice guidelines. This study is registered in ClinicalTrials.gov database, reference NCT02405507, and followed the CONSORT reporting guidelines (see online supplementary table S1).

\section{Patient and public involvement}

Before recruitment, volunteers that did not participate in the study were invited to be involved in the development of the bread formula used in this trial. They had no role in setting the research question, the outcome measures, the design or implementation of the study. We included inputs from the participants of the study, namely any burden associated with the procedures, throughout the follow-up in order to optimise their involvement and compliance. Participants were involved in the sensory evaluation of the study breads, as well as in the recruitment process by encouraging others to participate. On publication, participants will be informed of the results of this study through direct email.

\section{Study design and intervention}

Our study was a 15-week, randomised, double-blinded, crossover, controlled clinical trial. The trial comprised four stages: a run-in period ( 2 weeks), two crossover intervention periods (4weeks each) and a washout period between interventions ( 5 weeks). At the end of each stage, blood samples for measurement of plasma cholesterol (total, low-density lipoprotein (LDL) cholesterol and high-density lipoprotein (HDL)), triglycerides, $\mathrm{C}$ reactive protein (CRP) and postprandial glucose were collected from each study participant as primary outcomes. Blood 
samples were also collected for measurement of fasting glucose and insulin as secondary outcomes (see online supplementary figure S1). Primary and secondary outcomes related with gastrointestinal discomfort were also evaluated in this trial; however, they will be reported later.

Participants were instructed not to change their physical activity levels, maintain their dietary habits and do not consume any food or dietary product supplemented with germ during the study. Compliance to the study protocol (daily consumption of bread) was monitored through daily self-reported questionnaire, since there is no biomarker for wheat germ intake. Participants were randomly assigned into two intervention groups (ratio 1:1) using a computer-generated allocation sequence by a statistician not involved in recruitment and intervention delivery. The intervention arm comprised the daily consumption of wheat bread $(100 \mathrm{~g})$ supplemented with wheat germ $(6 \mathrm{~g})$, whereas the control arm involved the daily intake of wheat bread $(100 \mathrm{~g})$ without any supplementation. The bread provided to participants replaced their usual bread intake during meals, namely during breakfast or afternoon snack.

The participants and the research team were blinded to the study breads (intervention/control). In this regard, we previously tested the best formula that masked wheat germ supplementation, in terms of bread texture, volume and flavour. Moreover, the bread was delivered to each participant in opaque bags with a label code (A/B); only the outsourced company responsible for bread production (Padaria Ribeiro Lda., Portugal) knew the correspondence code. The unblinding was performed after the statistical analysis had been completed.

\section{Bread formulation}

Control breads were prepared by mixing $6 \mathrm{~kg}$ refined wheat flour (Germen S.A., Portugal) with 3.3L water, $97 \mathrm{~g}$ of salt, $60 \mathrm{~g}$ of bread improver mix (Germen, S.A., Portugal) and $180 \mathrm{~g}$ of baker's yeast. The mixture then fermented for $15 \mathrm{~min}$ at room temperature. Dough was divided and moulded into pieces (123 g each), and then placed in a fermentation chamber with $80 \%$ relative humidity for $60 \mathrm{~min}$ at $30^{\circ} \mathrm{C}$. Finally, baking was done at $190^{\circ} \mathrm{C}$ during $20 \mathrm{~min}$. Preparation of intervention bread was similar to control bread; $480 \mathrm{~g}$ of refined wheat flour was replaced by raw wheat germ (Germen S.A., Portugal) only.

Nutrient composition of control and intervention breads was analysed by Silliker Portugal, S.A. (Mérieux Nutrisciences Corporation).

\section{Blood sampling and analysis}

The outcomes variables were measured on study participants under 12 hours overnight fasting conditions, at the end of (1) run-in, (2) first intervention, (3) washout and (4) second intervention. Accordingly, a venous blood sample was collected by venipuncture into serum separator tubes (BD Vacutainer SST II Advance, Becton,
Dickinson and Company). For measuring glycated haemoglobin (HbAlc), blood was collected into tubes containing $\mathrm{K}_{2}$ EDTA (BD Vacutainer; Becton, Dickinson and Company). All venous blood samples were centrifuged at $1377 \times \mathrm{g}$ for $10 \mathrm{~min}$ at room temperature (CompactStar CS4; VWR), within 30 min after collection. A serum aliquot was immediately stored in a special cool transport container $\left(\right.$ at $<-10^{\circ} \mathrm{C}$ ) for insulin quantification. All biological samples were shipped to an outsourced certified medical laboratory (Clínica Laboratorial de Guimarães, S.A., Portugal) under refrigerated conditions. Analysis was performed within 24 hours after collection. Fasting glucose, total cholesterol and triglycerides were measured using specific enzymatic colorimetric methods, whereas LDL and HDL cholesterol were quantified by the elimination/catalase method. Serum CRP concentrations were measured by latex-enhanced immunoturbidimetric assay. These biochemical quantifications were done with the automatic analyser ADVIA 1800 (Siemens Healthcare Diagnostics). Insulin was determined by chemiluminescent microparticle immunoassay method using the automatic analyser Architect i2000 (Abbott Laboratories). The insulin resistance was estimated using the homeostasis model assessment for insulin resistance (HOMAIR $)^{35}{ }^{36}$ : (fasting insulin $(\mu \mathrm{U} / \mathrm{mL}) \times[$ fasting glucose $(\mathrm{mg} /$ dL) $\div 18.01]) / 22.5$

Regarding the postprandial glucose, four capillary blood samples were obtained by finger prick sampling using disposable lancet devices (Glucocard MX; Arkray) and a glucose metre. Postprandial glycaemia was measured in the fasting state $(0)$ and at 30,60 and 120 min after intervention or control bread intake. The postprandial glucose response was expressed as the incremental area under curve (IAUC), by using the trapezoidal rule ignoring the area below the fasting baseline, as previously described. ${ }^{37}$

\section{Statistical analysis}

Statistical analysis was performed using SPSS V.23 software (SPSS, Chicago, Illinois, USA). Data from all participants who were randomly assigned and completed an initial assessment were included in the intention-totreat statistical analysis. Additionally, an analysis was also performed including the participants who adhered to the study protocol only, namely those that completed the outcome measures and complied with the daily bread intake (assessed by daily questionnaire). Numerical data are expressed as means $\pm \mathrm{SD}$, and treatment effects with $95 \%$ CI. Statistical significance was set at a two-sided $\mathrm{p}$ value of 0.05 . However, we also decided to perform an adjustment for multiple comparison because of the multiple primary outcomes analysed; thus, the type 1 error and significance level associated with any individual variable difference took into account all comparisons performed and were ruled significant after adjusting for the overall false discovery rate, using the Benjamini-Hochberg procedure (with $\left.\mathrm{q}^{*}=0.05\right) .{ }^{38}$

Intervention effects were calculated as the difference between the change during each 4-week intervention 


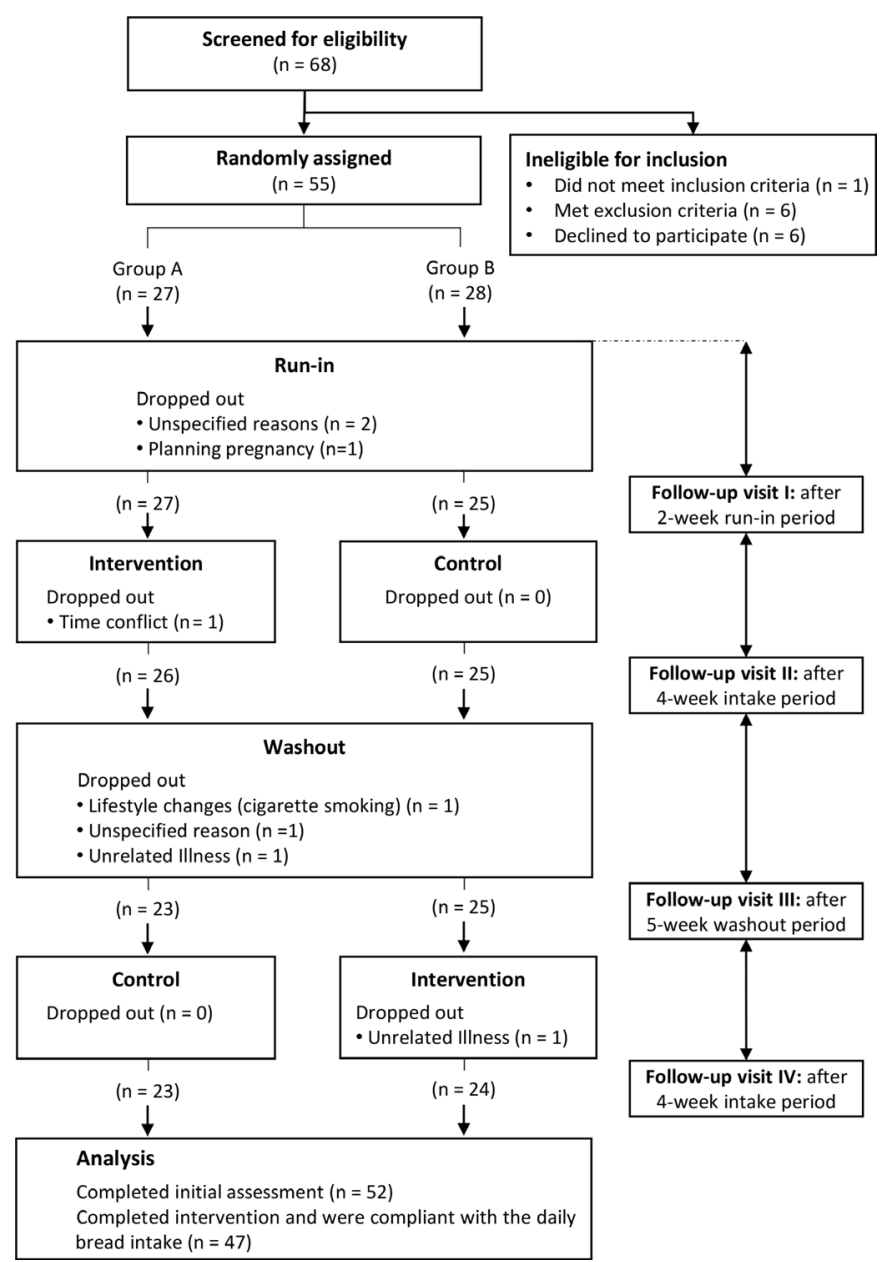

Figure 1 Flow chart of participants through the study.

period and the change during the 4-week control period. A linear mixed model for repeated measures, with compound symmetry as the covariance structure, was used to determine whether the intervention effects were statistically significant. Compound symmetry was used, instead of the autoregressive or unstructured structure because it resulted in the best fit according to a likelihood ratio test. Intervention, period and sequence were included as fixed variables. In order to account for between subject variability and to adjust for any non-specific differences, subjects were included as random effects. We also included intervention-sequence interaction as a fixed effect in the model to assess potential carryover effects. When carryover was significant, we reported the estimated intervention effect for each sequence and in these cases, we only used the first period of the crossover trial in the analysis, following Pocock's recommendations. ${ }^{39}$

\section{RESULTS}

\section{Baseline characteristics}

Of 68 subjects screened to assess eligibility to participate in the study protocol, 55 were randomly assigned: 27 to the intervention-control sequence and 28 to the controlintervention sequence. Fifty-two participants completed
Table 1 Baseline characteristics of study participants included in the analysis*

\begin{tabular}{|c|c|c|}
\hline Characteristics & $\mathbf{N}$ & Value \\
\hline \multicolumn{3}{|l|}{ Sex, n } \\
\hline Male & & 16 \\
\hline Female & & 36 \\
\hline Age, years & 52 & $33.67 \pm 11.69$ \\
\hline Body weight, kg & 52 & $66.69 \pm 11.84$ \\
\hline $\mathrm{BMI}, \mathrm{kg} / \mathrm{m}^{2}$ & 52 & $23.98 \pm 3.98$ \\
\hline Total cholesterol, mg/dL & 52 & $173.73 \pm 32.17$ \\
\hline HDL cholesterol, mg/dL & 52 & $60.71 \pm 15.04$ \\
\hline LDL cholesterol, mg/dL & 52 & $96.15 \pm 26.33$ \\
\hline Triglycerides, mg/dL & 52 & $84.40 \pm 56.42$ \\
\hline Glucose, mg/dL & 52 & $83.87 \pm 6.44$ \\
\hline Insulin, $\mu \mathrm{U} / \mathrm{mL}$ & 50 & $7.15 \pm 3.56$ \\
\hline HOMA-IR & 50 & $1.49 \pm 0.79$ \\
\hline $\mathrm{HbA1c}, \%$ & 51 & $5.17 \pm 0.25$ \\
\hline IAUC glucose, mg.min/dL & 50 & $3322.24 \pm 2086.88$ \\
\hline CRP, mg/dL & 52 & $0.12 \pm 0.18$ \\
\hline
\end{tabular}

${ }^{*}$ Mean \pm SD.

CRP, C reactive protein; HbA1c, glycated haemoglobin; HDL, highdensity lipoprotein; HOMA-IR, homeostasis model assessment for insulin resistance; IAUC, incremental area under the curve: LDL, low-density lipoprotein.

an initial assessment and were included in the statistical analysis (figure 1). Eight individuals dropped out: three did not wish to continue the study for unspecified reasons, three declined to participate due to personal reasons (see figure 1) and the last two because of unrelated illness (gastroparesis and pneumonia). Study participants were healthy men and women, with a mean age of 34 years (range: $18-59$ years), and body mass index (in $\mathrm{kg} / \mathrm{m}^{2}$ ) between 19 and 38 (34 normal weight, 14 overweight and four obese). All participants had normal fasting glucose and normal glucose tolerance. At baseline, $42 \mathrm{had}$ normal fasting total plasma cholesterol $(<200 \mathrm{mg} / \mathrm{dL})$, eight had borderline-high fasting total plasma cholesterol (200-239 mg/dL) and two had high fasting total plasma cholesterol $(>240 \mathrm{mg} / \mathrm{dL})$. Fifty participants had normal fasting total plasma triglycerides $(<150 \mathrm{mg} / \mathrm{dL})$ and two had high fasting total plasma triglycerides (200-499 mg/ $\mathrm{dL}$ ). Test results for haemoglobin and for liver function showed no evidence of ill health. Baseline characteristics of the 52 study participants that were included in the intention-to-treat statistical analysis are listed in table 1.

\section{Participant compliance}

Daily questionnaires revealed good compliance to the study protocol. The daily consumption of bread was assessed and the average compliance was $92.1 \% \pm 9.3$ and did not vary depending of bread type. Intervention and control arm had the same percentage of compliance: 


\begin{tabular}{|c|c|c|}
\hline & $\begin{array}{l}\text { Wheat germ- } \\
\text { enriched bread }\end{array}$ & Control bread \\
\hline Energy, kJ & 1154.7 & 1182.8 \\
\hline Protein, $\mathrm{g}$ & 9.7 & 8.8 \\
\hline Fat, g & 5.4 & 5.5 \\
\hline Carbohydrate, $g$ & 44.4 & 47.0 \\
\hline Dietary fibre, $\mathrm{g}$ & 4.3 & 4.0 \\
\hline Total sugar, g & 3.4 & 3.4 \\
\hline Starch, g & 40.8 & 43.4 \\
\hline Total phytosterols, mg & 52.0 & 30.0 \\
\hline Moisture, $g$ & 34.5 & 32.9 \\
\hline Ash, g & 1.7 & 1.9 \\
\hline \multicolumn{3}{|c|}{ Fatty acid, \% of total fatty acids } \\
\hline $14: 0$ & 0.2 & 0.0 \\
\hline 16:0 & 18.9 & 20.7 \\
\hline $16: 1$ & 3.3 & 3.7 \\
\hline 18:0 & 2.5 & 4.6 \\
\hline $18: 1 n-9$ & 14.9 & 16.0 \\
\hline $18: 1 n-7$ & 1.2 & 1.1 \\
\hline $18: 2 n-6$ & 52.9 & 49.8 \\
\hline $20: 0$ & 0.2 & 0.0 \\
\hline $18: 3 n-3$ & 5.1 & 3.6 \\
\hline $20: 1 n-9$ & 0.8 & 0.5 \\
\hline
\end{tabular}

$92.2 \% \pm 11.1 \%$ and $92.0 \% \pm 10.0$, respectively $(\mathrm{p}=0.920)$. None of the participants reported adverse effects.

\section{Composition of intervention and control breads}

The content of protein and dietary fibre was higher in the wheat germ-enriched bread $(9.6 \%$ and $7.5 \%$, respectively), whereas control bread had a higher content of carbohydrate and starch (5.5\% and $6.0 \%$, respectively). The content of fat and energy are comparable in both breads. As expected, the quantity of total phytosterols and alpha-linolenic acid was $73.3 \%$ and $41.7 \%$ higher in the intervention bread, respectively (table 2 ).

\section{Blood lipids}

There was no statistically significant difference between wheat germ-enriched and control breads, after 4-week intake, for cholesterol (total, LDL and HDL) and serum triglycerides (table 3 ).

No difference was also observed for those participants who completed the outcome measures and complied with the daily bread intake between wheat germ-enriched bread and control bread $(n=47$; total cholesterol: $\mathrm{p}=0.797$, HDL cholesterol: $\mathrm{p}=0.996$, LDL cholesterol: $\mathrm{p}=0.665$ and triglycerides: $\mathrm{p}=0.762$ ). The same result was obtained when participants with normal fasting total plasma cholesterol and triglycerides are analysed $(n=41$; total cholesterol: $\mathrm{p}=0.981$, HDL cholesterol: $\mathrm{p}=0.413$, LDL cholesterol: $\mathrm{p}=0.833$ and triglycerides: $\mathrm{p}=0.718$ ).

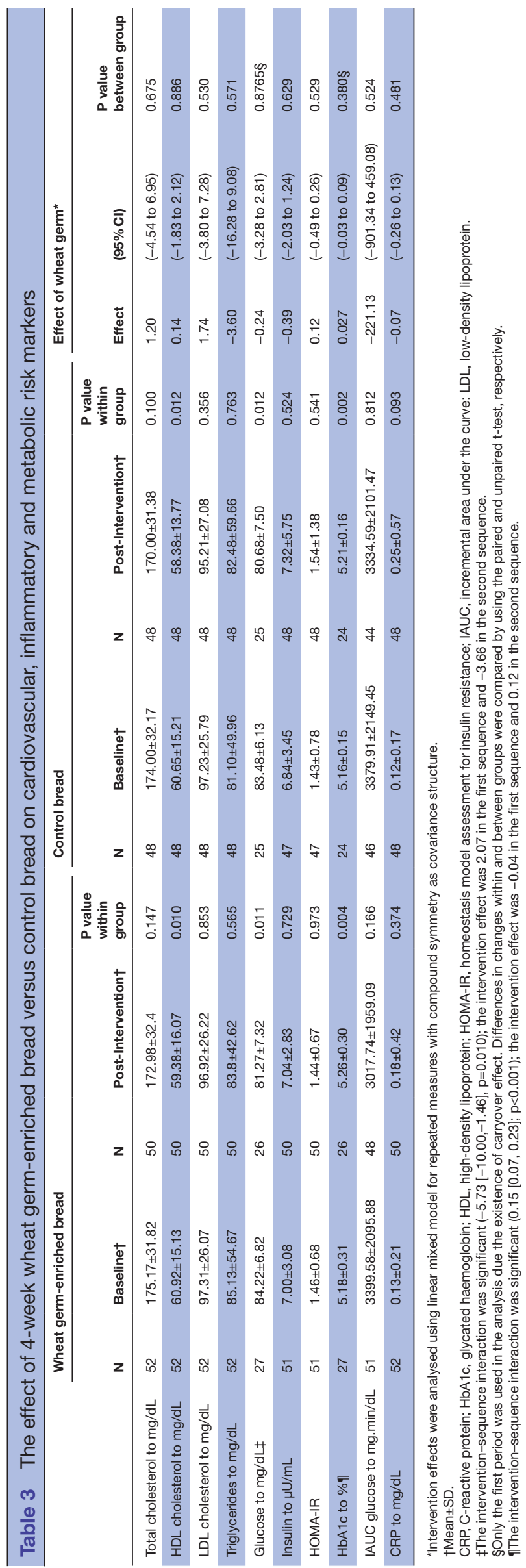



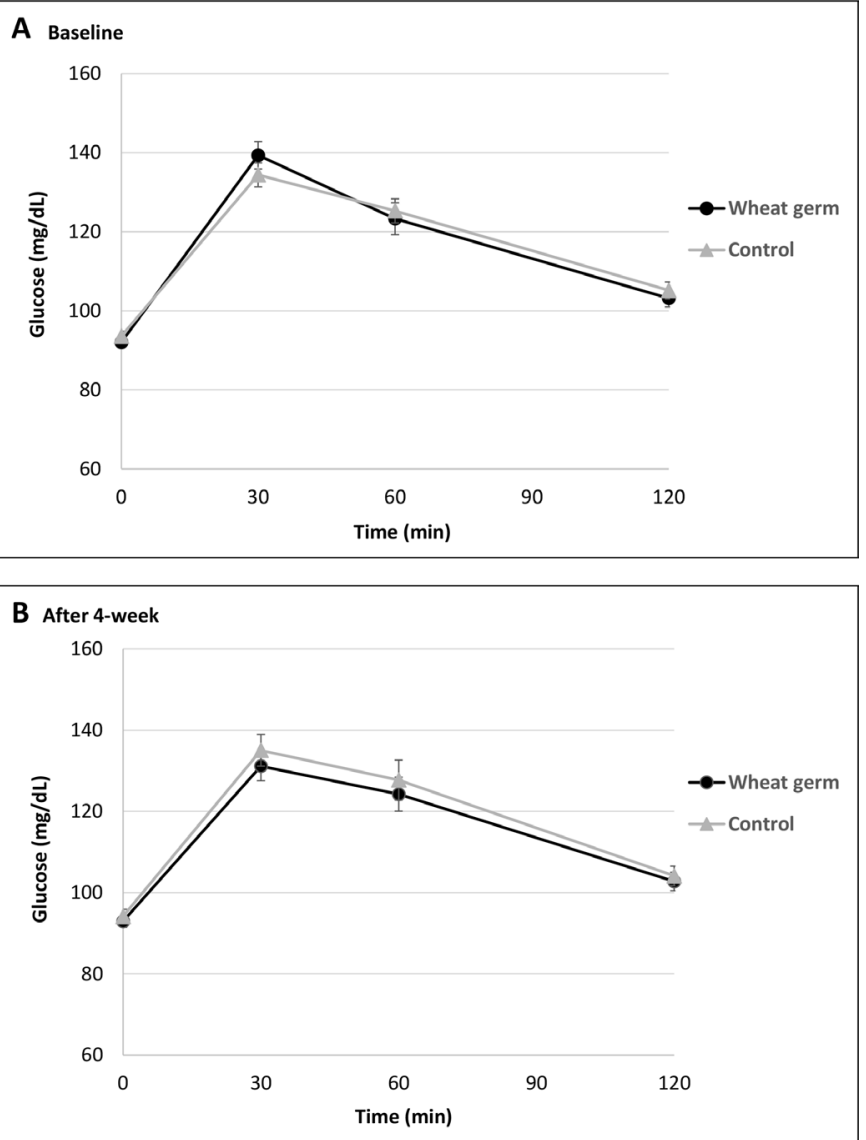

Figure 2 Mean \pm SEM postprandial glucose concentrations in response to a $100 \mathrm{~g}$ wheat germ-enriched bread or control bread, at baseline (A), and after 4 week intervention (B). No significant effect in the incremental area under the curve was observed between wheat germ-enriched and control breads $(p=0.524)$.

The results were not statistically different when participants with borderline or high fasting total plasma cholesterol were included $(\mathrm{n}=10$; total cholesterol: $\mathrm{p}=0.432$, HDL cholesterol: $\mathrm{p}=0.170$, LDL cholesterol: $\mathrm{p}=0.781$ and triglycerides: $p=0.508$ ). Statistical analysis of blood lipid outcomes showed no interaction between intervention and sequence, potentially excluding a carryover effect.

\section{Glucose metabolism and CRP}

There were no significant changes in postprandial glucose peak values (at $30 \mathrm{~min}$ ) after 4-week consumption of wheat germ-enriched bread $(-5.13 \pm 3.03 \mathrm{mg} / \mathrm{dL}$; $\mathrm{p}=0.097)$ or control bread $(2.35 \pm 3.73 \mathrm{mg} / \mathrm{dL} ; \mathrm{p}=0.531)$, and neither between interventions $(\mathrm{p}=0.182)$. Blood glucose response curves for 2 hours after the intake of intervention and control breads are shown in figure 2.

Comparing with control, wheat germ-enriched bread had no significant effect on IAUC glucose, fasting insulin or HOMA-IR (table 3), suggesting that $6 \mathrm{~g}$ of wheat germ did not improve postprandial glycaemia or glucose metabolism. No differences in CRP were observed between wheat germ-enriched and control breads. CRP and glucose results are not statistically different when only participants who adhered to the study protocol were analysed ( $n=47$; IAUC: $p=0.597$, insulin: $p=0.709$, HOMAIR: $p=0.597$, and CRP: $p=0.959$ ).

The intervention-sequence interaction was significant for fasting glucose $(-5.73[-10.00 ;-1.46], \mathrm{p}=0.010)$ and for HbA1c $(0.15$ [0.07; 0.23], $\mathrm{p}<0.001)$, revealing the existence of a carryover effect in these two outcomes. Therefore, the crossover design was not considered and only the first period was used in the analysis, following Pocock's recommendations. ${ }^{39}$ Nevertheless, no differences in fasting glucose or HbAlc between wheat germ-enriched and control breads were observed. HbA1c reflects the average blood glucose level in previous 2 to 3 months. ${ }^{40}$

\section{DISCUSSION}

In the present study, we evaluated the health effects of wheat germ intake in 52 healthy individuals. Blood lipids (cholesterol and triglycerides), fasting and postprandial glucose, CRP and insulin were evaluated during 15 weeks in a randomised crossover design. The results presented show that the intake of $6 \mathrm{~g} /$ day of wheat germ for 4 weeks has no effect on cholesterol and triglycerides levels, on glucose metabolism, namely in IAUC glucose, and on insulin sensitivity (HOMA-IR). Thus, our findings strongly suggest that wheat germ by itself has no impact on the cardiovascular risk factors evaluated and, thus, a beneficial physiological effect of wheat germ up to $6 \mathrm{~g}$ / day, by decreasing the risk of CVD in normal adults, is unlikely. Six grams of wheat germ intake per day corresponds to 2.4-fold increase in the germ present in the daily recommendation for whole grains intake and to a sixfold increase in the global mean consumption of germ.

In this study, we elucidated the role of germ in the primary prevention of cardiovascular events, and for this, we tested and developed a wheat germ-enriched bread without compromising the nutritional status (table 2), texture and flavour (see online supplementary table S2). Importantly, besides allowing blinding and the analysis of the effect of added germ individually, this no-difference is crucial for consumer's acceptance if this is envisioned as a long-term goal. Bread was chosen as vehicle for germ intake because it is a staple food, major contributor to carbohydrates intake, whereas the wheat is globally a staple grain. Up until now, the beneficial effect of wheat germ in lowering blood cholesterol and triglycerides was only demonstrated when used as a diet supplementation with $20 \mathrm{~g}^{24}$ or $30 \mathrm{~g} / \mathrm{day}^{23}$ during 4 -week periods. Incorporation of such high proportions of wheat germ in food products, without impairing their nutritional profiles, is difficult since germ changes their sensory proprieties. Our study is the first interventional study evaluating the effect of wheat germ in higher doses than the global mean consumption of germ and higher than the germ present in the recommended doses of whole grains. This intervention is a randomised, double-blinded, controlled clinical trial that uses a functional food product as supplementation vehicle, and not a dietary supplement. Our study 
followed the best practices for designing, conducting and reporting clinical trials to support health claims on food products, namely random allocation, double blinding, reporting methods to measure and maximise compliance. The strength of the current study also includes the use of validated outcomes which are considered to be beneficial physiological effects for human health. ${ }^{34}$ Finally, to our knowledge, this is the largest study to assess the impact of germ intake in human subjects and, importantly, targets the general population instead of a high-risk group. The limitations of this study include the duration of the intervention period. Although 4 weeks is considered the minimal intervention for evaluating an effect on lipoprotein cholesterol, 8 weeks would be more desirable; however, such intervention period in a crossover study could have a major impact on loss to follow-up. Second, the absence of a biomarker specific for germ intake is also a limitation; adherence was monitored through daily self-reported questionnaire and, though compliance with the study protocol was optimal, it is uncertain whether there was over-reporting. Third, the presence of carryover effects in the fasting glucose and HbAlc reduced the statistical power for these two outcomes, since the crossover design was not considered and only the first period was analysed. Lastly, we decided not to collect information about diet and physical activity levels during the study in order to avoid changes in general participants' lifestyle and dietary patterns, and this could be seen as a limitation.

In order to correctly inform consumers and food and nutrition policy makers about the benefits of supplementing food products with whole grain ingredients, there is an urgent need for clarifying the differences (whether any) between whole grains and bran or germ individually. First, adding bran or germ individually may not have identical physiological benefits as the whole grain; and, second, these two fractions alone may have distinct physiological effects on cardiovascular health promotion when compared with the whole grain. ${ }^{41}$ Germ of wheat was chosen due to its potential to reduce blood cholesterol and triglycerides concentrations, as suggested in previous studies. ${ }^{23}{ }^{24}$ The proposed mechanisms for lowering plasma cholesterol include (1) the inhibition of pancreatic lipase activity by soluble proteins present on wheat germ, ${ }^{42}$ (2) the reduction in triglyceride lipolysis, ${ }^{43}$ and (3) reduction in cholesterol absorption by the endogenous wheat germ phytosterols. ${ }^{44}$ However, the existing evidence regarding the lipid-lowering properties of wheat germ from previous studies is controversial. First, reduction of total cholesterol and LDL was only evaluated in hypercholesterolaemic individuals after diet supplementation with $20 \mathrm{~g} /$ day for a 4 -week period. Second, daily ingestion of $30 \mathrm{~g}$ wheat germ supplement for 4 weeks markedly decreases $(-39 \%)$ plasma triglycerides in hypertriglyceridaemic individuals, whereas no reduction was observed in the normotriglyceridaemic subgroup. ${ }^{23}$

In our study, we enrolled a moderately large and heterogeneous sample of participants which is representative of general population, with normal, borderline-high and high fasting total plasma cholesterol and triglycerides. The statistical power of this study is higher than preceding studies and, for that reason, smaller differences could be detected if they indeed existed. Thus, it is not plausible that the absence of any statistical significance on the wheat germ-induced changes is due to the lack of statistical power. However, the inexistence of wheat germ effects reported here does not necessarily disprove the potential cholesterol-lowering and triglyceride-lowering effect described in previous studies, in particular, because in those studies, wheat germ was consumed in higher doses and by individuals at risk. In our opinion, a doseresponse analysis for wheat germ intake and lipid profile should be investigated in the future, for general population and high-risk groups.

Effect of wheat germ in improving postprandial glycaemia or glucose metabolism was not demonstrated in our study, in accordance with previous studies. Earlier studies addressing the long-term impact of wheat germ on blood lipids of hypercholesterolaemic individuals did not observe any significant variation on fasting glucose, insulin, fructosamine and $\mathrm{HbAlc}^{24}$; and one single study with six healthy participants showed that wheat germ had no effect on postprandial glucose and insulin concentrations. ${ }^{45}$ Even so, we decided to investigate the impact of wheat germ on glucose metabolism because it was recently suggested that intake of alpha-linolenic acid has been associated with lower insulin resistance ${ }^{46}$ and our wheat germ enriched-bread contains more $41.7 \%$ of alpha-linolenic than control bread (table 2).

In summary, our goal was to demonstrate the effect of a staple food supplemented with wheat germ in its maximal concentration without compromising its sensory properties. However, the intake of $6 \mathrm{~g} /$ day of wheat germ does not contribute to reduce the cardiovascular risk factors plasma triglycerides, total cholesterol, LDL cholesterol, or increase the HDL cholesterol levels, or even improve glycaemic control, in a generally healthy normal population. In order to justify an enrichment of food products with wheat germ as a public health approach to prevent CVD, the beneficial effects of wheat germ on human health should be investigated in other food vehicles that can accommodate higher doses of germ. Chemical and sensory proprieties of biscuits, noodles and cakes supplemented with increasing amounts of wheat germ (up to $30 \%$ ) have been recently analysed and improved. ${ }^{32} 4748$ These technological advances let us envisage that other food products functionalised with higher wheat germ content can be developed. Future studies following our clinical trial design are needed to elucidate if high amounts of daily intake of wheat germ are effective in reducing CVD risk.

\section{Author affiliations}

${ }^{1}$ Centre for Health Technology and Services Research (CINTESIS), Porto, Portugal ${ }^{2}$ Department of Community Medicine, Information and Health Decision Sciences (MEDCIDS), Universidade do Porto, Porto, Portugal 
${ }^{3}$ Nutrition \& Metabolism, NOVA University of Lisbon, NOVA Medical School, Lisbon, Portugal

${ }^{4}$ Infectious Diseases Service, Hospital Curry Cabral, Lisboa, Portugal

${ }^{5}$ Center of Biological Engineering, University of Minho, Braga, Portugal

Acknowledgements We thank Diana Sintra, Francisca Mendes and Sónia Norberto for their assistance during follow-up. We are also especially grateful to all subjects for their participation in this study.

Contributors AM-R was partially responsible for study design, statistical analysis plan, carrying out the trial, manuscript writing and final revision. HP was partially responsible for study design, carrying out the trial, manuscript writing and final revision. CM collaborated in the study design, data collection and final revision of the manuscript. JAT collaborated in the study design and final revision of the manuscript. CC was responsible for the general coordination of the project, study design, manuscript writing and final revision. LFA was responsible for the general coordination of the project, study design, statistical analysis plan, manuscript writing and final revision.

Funding This work was supported by the Programa Operacional Competitividade e Internacionalização-COMPETE2020 and by National Funds through Fundação para a Ciência e a Tecnologia-FCT (through the operations FCOMP-01-0202FEDER-038861 and POCI-01-0145-FEDER-007746).

Disclaimer The COMPETE2020 and FCT had no role in the design, analysis or writing of this article.

Competing interests None declared.

Patient consent Obtained.

Ethics approval Health Ethics Committee of São João Hospital Centre and the Ethics Committee of the Faculty of Medicine of the University of Porto.

Provenance and peer review Not commissioned; externally peer reviewed.

Data sharing statement The full data sets generated during and/or analysed during the current study are not publicly available because the ethics committee only allowed the use of the data in the context of the present research project; however, anonymised partial data sets or summaries of the data are available from the corresponding author on reasonable request.

Open access This is an open access article distributed in accordance with the Creative Commons Attribution Non Commercial (CC BY-NC 4.0) license, which permits others to distribute, remix, adapt, build upon this work non-commercially, and license their derivative works on different terms, provided the original work is properly cited, appropriate credit is given, any changes made indicated, and the use is non-commercial. See: http://creativecommons.org/licenses/by-nc/4.0/.

\section{REFERENCES}

1. World Health Organization. Top 10 causes of death worldwide. 2017. http://www.who.int/mediacentre/factsheets/fs310/en/

2. Ferns GA. New and emerging risk factors for CVD. Proc Nutr Soc 2008;67:223-31.

3. Ezzati M, Obermeyer Z, Tzoulaki I, et al. Contributions of risk factors and medical care to cardiovascular mortality trends. Nat Rev Cardiol 2015;12:508-30.

4. Lin JS, O'Connor E, Evans CV, et al. Behavioral counseling to promote a healthy lifestyle in persons with cardiovascular risk factors: a systematic review for the U.S. Preventive Services Task Force. Ann Intern Med 2014;161:568-78.

5. Bechthold A, Boeing $\mathrm{H}$, Schwedhelm $\mathrm{C}$, et al. Food groups and risk of coronary heart disease, stroke and heart failure: A systematic review and dose-response meta-analysis of prospective studies. Crit Rev Food Sci Nutr 2017:1-20.

6. Chen GC, Tong X, Xu JY, et al. Whole-grain intake and total, cardiovascular, and cancer mortality: a systematic review and metaanalysis of prospective studies. Am J Clin Nutr 2016;104:164-72.

7. Aune D, Keum N, Giovannucci E, et al. Whole grain consumption and risk of cardiovascular disease, cancer, and all cause and cause specific mortality: systematic review and dose-response metaanalysis of prospective studies. BMJ 2016;353:i2716.

8. Zong G, Gao A, Hu FB, et al. Whole grain intake and mortality from all causes, cardiovascular disease, and cancer: a meta-analysis of prospective cohort studies. Circulation 2016;133:2370-80.

9. Ye EQ, Chacko SA, Chou EL, et al. Greater whole-grain intake is associated with lower risk of type 2 diabetes, cardiovascular disease, and weight gain. J Nutr 2012;142:1304-13.
10. Wei H, Gao Z, Liang R, et al. Whole-grain consumption and the risk of all-cause, CVD and cancer mortality: a meta-analysis of prospective cohort studies - CORRIGENDUM. Br J Nutr 2016;116:952.

11. Jacobs DR, Andersen LF, Blomhoff R. Whole-grain consumption is associated with a reduced risk of noncardiovascular, noncancer death attributed to inflammatory diseases in the lowa Women's Health Study. Am J Clin Nutr 2007;85:1606-14.

12. Lupton JR, Robinson MC, Morin JL. Cholesterol-lowering effect of barley bran flour and oil. J Am Diet Assoc 1994;94:65-70.

13. Behall KM, Scholfield DJ, Hallfrisch J. Diets containing barley significantly reduce lipids in mildly hypercholesterolemic men and women. Am J Clin Nutr 2004;80:1185-93.

14. Dubois C, Armand M, Senft M, et al. Chronic oat bran intake alters postprandial lipemia and lipoproteins in healthy adults. Am J Clin Nutr 1995;61:325-33.

15. Charlton KE, Tapsell LC, Batterham MJ, et al. Effect of 6 weeks consumption of $\beta$-glucan-rich oat products on cholesterol levels in mildly hypercholesterolaemic overweight adults. Br J Nutr 2012:107:1037-47.

16. de Munter JS, Hu FB, Spiegelman D, et al. Whole grain, bran, and germ intake and risk of type 2 diabetes: a prospective cohort study and systematic review. PLoS Med 2007;4:e261.

17. Jensen MK, Koh-Banerjee P, Hu FB, et al. Intakes of whole grains, bran, and germ and the risk of coronary heart disease in men. $A m \mathrm{~J}$ Clin Nutr 2004;80:1492-9.

18. Jensen MK, Koh-Banerjee P, Franz M, et al. Whole grains, bran, and germ in relation to homocysteine and markers of glycemic control, lipids, and inflammation 1. Am J Clin Nutr 2006;83:275-83.

19. Wu H, Flint AJ, Qi Q, et al. Association between dietary whole grain intake and risk of mortality: two large prospective studies in US men and women. JAMA Intern Med 2015;175:373-84.

20. Lairon D, Lacombe $C$, Borel $P$, et al. Beneficial effect of wheat germ on circulating lipoproteins and tissue lipids in rats fed a high fat, cholesterol-containing diet. J Nutr 1987;117:838-45.

21. Cara L, Borel P, Armand M, et al. Effects of increasing levels of raw or defatted wheat germ on liver, feces and plasma lipids and lipoproteins in the rat. Nutr Res 1991;11:907-16.

22. Borel P, Lairon D, Senft M, et al. Wheat bran and wheat germ: effect on digestion and intestinal absorption of dietary lipids in the rat. Am J Clin Nutr 1989;49:1192-202.

23. Cara L, Borel P, Armand M, et al. Plasma lipid lowering effects of wheat germ in hypercholesterolemic subjects. Plant Foods Hum Nutr 1991:41:135-50.

24. Cara L, Armand M, Borel P, et al. Long-term wheat germ intake beneficially affects plasma lipids and lipoproteins in hypercholesterolemic human subjects. J Nutr 1992;122:317-26.

25. Micha R, Khatibzadeh S, Shi P, et al. Global, regional and national consumption of major food groups in 1990 and 2010: a systematic analysis including 266 country-specific nutrition surveys worldwide. BMJ Open 2015;5:e008705.

26. Burton PM, Monro JA, Alvarez L, et al. Glycemic impact and health: new horizons in white bread formulations. Crit Rev Food Sci Nutr 2011:51:965-82.

27. Mattei J, Malik V, Wedick NM, et al. Reducing the global burden of type 2 diabetes by improving the quality of staple foods: The Global Nutrition and Epidemiologic Transition Initiative. Global Health 2015;11:23

28. Seal CJ, Nugent AP, Tee ES, et al. Whole-grain dietary recommendations: the need for a unified global approach. Br J Nutr 2016;115:2031-8.

29. Fardet A. New hypotheses for the health-protective mechanisms of whole-grain cereals: what is beyond fibre? Nutr Res Rev 2010;23:65-134.

30. Sjövall O, Virtalaine T, Lapveteläinen A, et al. Development of rancidity in wheat germ analyzed by headspace gas chromatography and sensory analysis. J Agric Food Chem 2000;48:3522-7.

31. González-Ferrero C, Sáiz-Abajo MJ. Characterization and stability studies of bioactive compounds and food matrices as evidence in support of health claims. Int J Food Sci Nutr 2015;66:S4-S12.

32. Aktaș $\mathrm{K}$, Bilgiçli $\mathrm{N}$, Levent $\mathrm{H}$. Influence of wheat germ and $\beta$-glucan on some chemical and sensory properties of Turkish noodle. J Food Sci Technol 2015;52:6055-60.

33. Li B, Zhao L, Chen $\mathrm{H}$, et al. Inactivation of lipase and lipoxygenase of wheat germ with temperature-controlled short wave infrared radiation and its effect on storage stability and quality of wheat germ oil. PLOS One 2016;11:e0167330.

34. Moreira-Rosário A, Pinheiro H, Calhau C, et al. Can wheat germ have a beneficial effect on human health? A study protocol for a randomised crossover controlled trial to evaluate its health effects. BMJ Open 2016;6:e013098. 
35. Matthews DR, Hosker JP, Rudenski AS, et al. Homeostasis model assessment: insulin resistance and beta-cell function from fasting plasma glucose and insulin concentrations in man. Diabetologia 1985;28:412-9.

36. Qi Q, Bray GA, Hu FB, Fb H, et al. Weight-loss diets modify glucosedependent insulinotropic polypeptide receptor rs2287019 genotype effects on changes in body weight, fasting glucose, and insulin resistance: the preventing overweight using novel dietary strategies trial. Am J Clin Nutr 2012;95:506-13.

37. Wolever TMS. The glycaemic index: a physiological classification of dietary carbohydrate. Oxfordshire: CABI, 2006.

38. Benjamini Y, Hochberg Y. Controlling the false discovery rate: a practical and powerful approach to multiple testing. Journal of the Royal Statistical Society: Series B 1995;57:289-300.

39. Pocock SJ. Clinical Trials: A Practical Approach. Chichester, UK: John Wiley \& Sons Ltd, 1983.

40. Zhong GC, Ye MX, Cheng JH, et al. HbA1c and risks of all-cause and cause-specific death in subjects without known diabetes: a dose-response meta-analysis of prospective cohort studies. Sci Rep 2016;6:24071.

41. Juan J, Liu G, Willett WC, et al. Whole grain consumption and risk of ischemic stroke: results from 2 prospective cohort studies. Stroke $2017 ; 48$
42. Lairon D, Borel P, Termine E, et al. Evidence for a proteinic inhibitor of pancreatic lipase in cereals, wheat bran and wheat germ. Nutr Rep Int 1985;32:1107-13.

43. Borel P, Martigne M, Senft M, et al. Effect of wheat bran and wheat germ on the intestinal uptake of oleic acid, monoolein, and cholesterol in the rat. J Nutr Biochem 1990;1:28-33.

44. Ostlund RE, Racette SB, Stenson WF. Inhibition of cholestero absorption by phytosterol-replete wheat germ compared with phytosterol-depleted wheat germ. Am J Clin Nutr 2003;77:1385-9.

45. Cara L, Dubois C, Borel P, et al. Effects of oat bran, rice bran, wheat fiber, and wheat germ on postprandial lipemia in healthy adults. Am J Clin Nutr 1992;55:81-8.

46. Heskey CE, Jaceldo-Siegl K, Sabaté J, et al. Adipose tissue $\alpha$-linolenic acid is inversely associated with insulin resistance in adults. Am J Clin Nutr 2016;103:1105-10.

47. Al-Marazeeq KM, Angor MM. Chemical characteristic and sensory evaluation of biscuit enriched with wheat germ and the effect of storage time on the sensory properties for this product. Food Nutr Sci 2017;08:189-95.

48. Levent H, Bilgiçli N. Quality evaluation of wheat germ cake prepared with different emulsifiers. J Food Qual 2013;36:334-41. 